\title{
Inner Diameter
}

National Cancer Institute

\section{Source}

National Cancer Institute. Inner Diameter. NCI Thesaurus. Code C101685.

The length of a straight line passing through the center of the inner open space of a circular or spheroid object that connects two points on the circumference. 